\title{
Three-dimensional granular contact dynamics with rolling resistance
}

\author{
J. Huang ${ }^{\mathrm{a}}$, M. Vicente da Silva ${ }^{\mathrm{a}, \mathrm{b}}$, K. Krabbenhoft ${ }^{\mathrm{a}, \mathrm{c}, *}$ \\ a Centre for Geotechnical Science and Engineering University of Newcastle, NSW, Australia \\ ${ }^{\mathrm{b}}$ Departamento de Engenharia Civil, Universidade Nova de Lisboa, Portugal \\ ${ }^{\mathrm{c}}$ Institute of Technology and Innovation, University of Southern Denmark, Odense, Denmark
}

\section{A R T I C L E I N F O}

\section{Article history:}

Received 29 June 2012

Received in revised form 24 August 2012

Accepted 29 August 2012

Available online 2 November 2012

\section{Keywords:}

Contact dynamics

Discrete element method

DEM

Rolling resistance

\begin{abstract}
A B S T R A C T
A three-dimensional granular contact dynamics scheme is presented. The scheme is variational in structure, thus making it possible to solve the governing equations by means of mathematical programming methods. To facilitate the modeling of natural grains using spherical geometries, a rolling resistance model is developed. A number of static and dynamic benchmark examples are considered including the granular column collapse problem where the agreement between simulation and previously published experimental results is found to be very good.
\end{abstract}

(c) 2012 Elsevier Ltd. All rights reserved.

\section{Introduction}

The motion and interaction of discrete particles can be simulated using one of two different methods. The most popular method is the discrete (or distinct) element method (DEM) pioneered by Cundall and Strack [7]. In this method, which is often referred to as a molecular dynamics (MDs) method, the positions of the particles are advanced in a fully explicit manner using sufficiently small time steps. Interaction between grains is accounted for by relating the overlap that may occur between particles to forces via an appropriate constitutive relation.

The second approach to the modeling of granular assemblies is the so-called non-smooth contact dynamics (CDs) method originally developed by Moreau and his co-workers [32-34,10]. In the most basic version of this method, the particles are considered perfectly rigid and the contact forces are determined as those that exactly prevent inter-particle penetration. An implicit time discretization is usually employed, thus allowing for larger time steps, and implying that collisions are 'smeared' over a finite time interval.

Although the MD method has been much more widely applied than the CD method, there are several examples of the application of the latter to the simulation of granular materials $[4,9,11,12,23$, 30,37,39-47,50-52].

Recently, a new formulation of the $C D$ method has been developed [17,15]. Besides making use of displacements as primary variables (where most other formulations make use of

\footnotetext{
* Corresponding author at: Centre for Geotechnical Science and Engineering University of Newcastle, NSW, Australia.

E-mail address: kristian.krabbenhoft@newcastle.edu.au (K. Krabbenhoft).
}

impulses), the formulation emphasizes a rigorous variational structure. This not only ensures existence and uniqueness of solutions $[48,38,2,17,15]$, but also paves the way for the application of efficient mathematical programming algorithms for the solution of the resulting problems. The present paper follows on from [17] by firstly extending the original two-dimensional scheme to three dimensions. Secondly, the inclusion of rolling resistance is detailed. This feature is essential in the idealization of natural grains as spheres. The resulting scheme is again variational in structure and the same algorithms as used in the two-dimensional case are applicable. In addition to these enhancements, the treatment of boundary conditions is discussed in some detail. Finally, the capabilities of the scheme are demonstrated by the solution of a number of problems with the primary aim of assessing the prospects of modeling natural, non-spherical grains using spherical grains that incorporate a finite amount of resistance to rolling.

\section{Governing equations}

\subsection{Equations of motion} by

The equations of motion for a single spherical particle are given

$m \dot{\boldsymbol{v}}(t)=\boldsymbol{f}_{\mathrm{ext}}$

$J \dot{\omega}(t)=\boldsymbol{m}_{\mathrm{ext}}$

where $\boldsymbol{v}(t)=\left[v_{x}(t), v_{y}(t), v_{z}(t)\right]^{\top}$ are the linear velocities, $\boldsymbol{\omega}(t)=$ $\left[\omega_{x}(t), \omega_{y}(t), \omega_{z}(t)\right]^{\top}$ are the angular velocities, $m$ is the mass, $J$ is the mass moment of inertia, and $\boldsymbol{f}_{\text {ext }}=\left(f_{x}, f_{y}, f_{z}\right)^{\top}$ ext and $\boldsymbol{m}_{\text {ext }}=$ $\left(m_{x}, m_{y}, m_{z}\right)^{\top}$ ext are external forces and moments respectively. 
Following [17], the $\theta$-method is used to discretize the equations of motion. Denoting the known position at $t_{0}$ by $\boldsymbol{x}_{0}$ and the unknown position at $t_{0}+\Delta t$ by $\boldsymbol{x}$, the time discrete linear momentum equations are given by

$\bar{m} \Delta \boldsymbol{x}=\overline{\boldsymbol{f}}_{0}$

$\boldsymbol{v}=\frac{1}{\theta}\left[\frac{\Delta \boldsymbol{x}}{\Delta t}-(1-\theta) \boldsymbol{v}_{0}\right]$

where $\Delta \boldsymbol{x}=\boldsymbol{x}-\boldsymbol{x}_{0}$ are the displacements and

$\bar{m}=\frac{1}{\theta \Delta t^{2}} m, \quad \overline{\boldsymbol{f}}_{0}=\boldsymbol{f}_{\mathrm{ext}}+\bar{m} \boldsymbol{v}_{0} \Delta t$

The angular momentum balance equations are discretized in an analogous manner to arrive at

$\bar{J} \Delta \boldsymbol{\alpha}=\overline{\boldsymbol{m}}_{0}$

$\omega=\frac{1}{\theta}\left[\frac{\Delta \boldsymbol{\alpha}}{\Delta t}-(1-\theta) \omega_{0}\right]$

where $\Delta \boldsymbol{\alpha}=\boldsymbol{\alpha}-\boldsymbol{\alpha}_{0}$ are the incremental rotation angles and

$\bar{J}=\frac{1}{\theta \Delta t^{2}} J, \quad \overline{\boldsymbol{m}}_{0}=\boldsymbol{m}_{\mathrm{ext}}+\bar{J} \omega_{0} \Delta t$

The stability properties of the $\theta$-method are well known [57]: for $\theta=\frac{1}{2}$ the an unconditionally stable and energy preserving scheme is recovered, for $\theta>\frac{1}{2}$ the scheme is unconditionally stable and dissipative, and for $\theta<\frac{1}{2}$ stability depends on the time step. In the context of collisions, the algorithmic energy dissipation that occurs for $\theta>\frac{1}{2}$ can be related to the physical dissipation associated with impact and thus to the restitution coefficient. Indeed, as shown in [17], a value of $\theta=\frac{1}{2}$ corresponds to an elastic collision while $\theta=1$ reproduces a perfectly inelastic collision.

\subsection{Frictional contact}

Consider the frictional contact between two spherical particles, $i$ and $j$, as shown in Fig. 1. Here and in the following, lower case superscripts refer to the particles while upper case superscripts refer to the contact. In terms of forces, the contact is defined by a set of self-equilibrated normal and two mutually orthogonal shear forces orthogonal to the particle normal. Let $\boldsymbol{n}_{0}^{I}$ be the outward normal of particle $i$ and let $\left\{\boldsymbol{n}_{0}^{I}, \boldsymbol{a}_{0}^{I}, \boldsymbol{b}_{0}^{I}\right\}$ be an orthogonal set of unit vectors. Incorporating contact forces, the time discrete linear momentum equations for the two particles can be expressed as

$\bar{m}^{i} \Delta \boldsymbol{x}^{i}+p^{I} \boldsymbol{n}_{0}^{I}+q_{a}^{I} \boldsymbol{a}_{0}^{I}+q_{b}^{I} \boldsymbol{b}_{0}^{I}=\overline{\boldsymbol{f}}_{0}^{i}$
$\bar{m}^{j} \Delta \boldsymbol{x}^{j}-p^{I} \boldsymbol{n}_{0}^{I}-q_{a}^{I} \boldsymbol{a}_{0}^{I}-q_{b}^{I} \boldsymbol{b}_{0}^{I}=\overline{\boldsymbol{f}}^{j}$

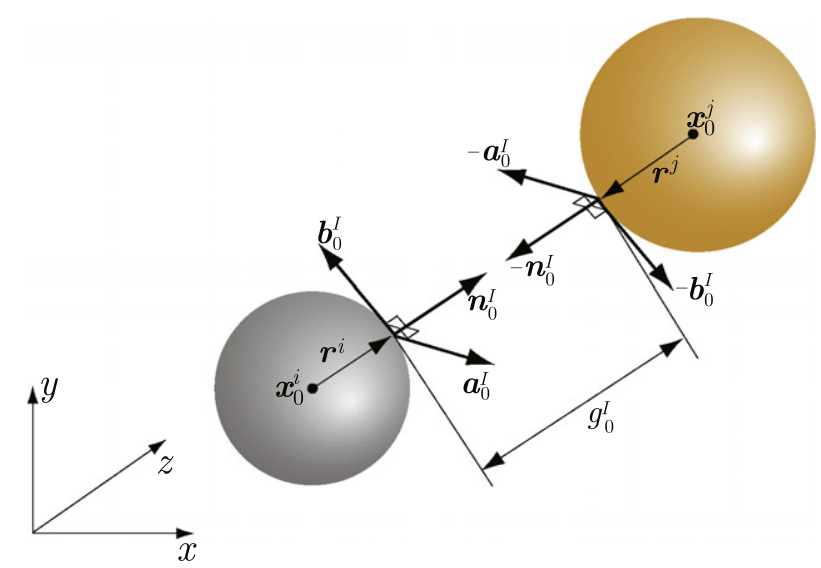

Fig. 1. Frictional interaction between two particles $i$ and $j$ at contact $I$. where $p^{I}$ is the normal force at the contact and $q_{a}^{I}$ and $q_{b}^{I}$ are the shear forces (see Fig. 1). These are all assumed positive opposite to the associated unit vectors, $\boldsymbol{n}_{0}^{I}, \boldsymbol{a}_{0}^{I}$ and $\boldsymbol{b}_{0}^{I}$ respectively. Similarly, the time discrete angular momentum equations are given by

$\bar{J}^{i} \Delta \boldsymbol{\alpha}^{i}-\boldsymbol{r}_{0}^{i} \times\left(q_{a}^{I} \boldsymbol{a}_{0}^{I}+q_{b}^{I} \boldsymbol{b}_{0}^{I}\right)=\overline{\boldsymbol{m}}_{0}^{i}$
$\bar{J}^{j} \Delta \boldsymbol{\alpha}^{j}+\boldsymbol{r}_{0}^{j} \times\left(q_{a}^{I} \boldsymbol{a}_{0}^{I}+q_{b}^{I} \boldsymbol{b}_{0}^{I}\right)=\overline{\boldsymbol{m}}_{0}^{j}$

or, alternatively, by

$\bar{J}^{i} \Delta \boldsymbol{\alpha}^{i}-\boldsymbol{R}_{0}^{i}\left(q_{a}^{I} \boldsymbol{a}_{0}^{I}+q_{b}^{I} \boldsymbol{b}_{0}^{I}\right)=\overline{\boldsymbol{m}}_{0}^{i}$

$\bar{J}^{j} \Delta \boldsymbol{\alpha}^{j}+\boldsymbol{R}_{0}^{j}\left(q_{a}^{I} \boldsymbol{a}_{0}^{I}+q_{b}^{I} \boldsymbol{b}_{0}^{I}\right)=\overline{\boldsymbol{m}}_{0}^{j}$

where

$\boldsymbol{R}^{\alpha}=\left[\begin{array}{ccc}0 & -r_{z}^{\alpha} & r_{y}^{\alpha} \\ r_{z}^{\alpha} & 0 & -r_{x}^{\alpha} \\ -r_{y}^{\alpha} & r_{x}^{\alpha} & 0\end{array}\right], \quad \alpha=i, j$

The rotations are here assumed positive following the left-hand rule (positive clockwise in 2D).

For general $n$-particle problems, it is convenient to introduce the following system matrices and arrays:

$\overline{\boldsymbol{M}}=\operatorname{diag}\left(\bar{m}^{1}, \bar{m}^{1}, \bar{m}^{1}, \ldots, \bar{m}^{n}, \bar{m}^{n}, \bar{m}^{n}\right)$

$\overline{\boldsymbol{J}}=\operatorname{diag}\left(\bar{J}^{1}, \bar{J}^{1}, \bar{J}^{1}, \ldots, \bar{J}^{n}, \bar{J}^{n}, \bar{J}^{n}\right)$

$\overline{\boldsymbol{f}}=\left(\overline{\boldsymbol{f}}^{1}, \ldots, \overline{\boldsymbol{f}}^{n}\right)^{\top}, \overline{\boldsymbol{m}}=\left(\overline{\boldsymbol{m}}^{1}, \ldots, \overline{\boldsymbol{m}}^{n}\right)^{\top}$

$\mathbf{g}=\left(g^{1}, \ldots, g^{N}\right)$

together with arrays of state variables:

$$
\begin{aligned}
& \boldsymbol{x}=\left(\boldsymbol{x}^{1}, \ldots, \boldsymbol{x}^{n}\right), \quad \boldsymbol{\alpha}=\left(\boldsymbol{\alpha}^{1}, \ldots, \boldsymbol{\alpha}^{n}\right) \\
& \boldsymbol{p}=\left(p^{1}, \ldots, p^{N}\right) \\
& \boldsymbol{q}^{I}=\left(q_{a}^{I}, q_{b}^{I}\right), \quad \boldsymbol{q}=\left(\boldsymbol{q}^{1}, \ldots, \boldsymbol{q}^{N}\right)
\end{aligned}
$$

The number of particles is here given by $n$ while $N$ is the number of contacts. Furthermore, introducing matrices $\boldsymbol{N}$ and $\widehat{\boldsymbol{N}}$ associated with the normal and shear forces, in the linear momentum balance Eq. (6) and a matrix $\boldsymbol{R}$ involving the local cross product matrices, $\boldsymbol{R}^{\alpha}$, of the angular momentum balance Eq. (8), the equations of motion can be written as

$\overline{\boldsymbol{M}} \Delta \boldsymbol{x}+\boldsymbol{N}_{0} \boldsymbol{p}+\widehat{\boldsymbol{N}}_{0} \boldsymbol{q}=\overline{\boldsymbol{f}}_{0}$
$\overline{\boldsymbol{J}} \Delta \boldsymbol{\alpha}-\boldsymbol{R}_{0} \boldsymbol{q}=\overline{\boldsymbol{m}}_{0}$

These equations are a direct extension of the equivalent twodimensional equations derived in [17], the only difference being the dimensionality of $\boldsymbol{q}$ and the associated system matrices.

\subsection{Variational formulation}

Following [17], the governing Eq. (12) can be cast in terms of the following optimization problem where Coulomb type friction conditions also have been included:

$$
\begin{array}{ll}
\underset{\boldsymbol{x}, \boldsymbol{\alpha}}{\operatorname{minimize}} \underset{\boldsymbol{p}, \boldsymbol{q}}{\operatorname{maximize}} & \frac{1}{2} \Delta \boldsymbol{x}^{\top} \overline{\boldsymbol{M}} \Delta \boldsymbol{x}+\frac{1}{2} \Delta \boldsymbol{\alpha}^{\top} \bar{J} \Delta \boldsymbol{\alpha} \\
& +\Delta \boldsymbol{x}^{\top}\left(\boldsymbol{N}_{0} \boldsymbol{p}+\widehat{\boldsymbol{N}}_{0} \boldsymbol{q}-\overline{\boldsymbol{f}}_{0}\right) \\
& +\Delta \boldsymbol{\alpha}^{\top}\left(-\boldsymbol{R}_{0} \boldsymbol{q}-\overline{\boldsymbol{m}}_{0}\right) \\
& -\boldsymbol{g}_{0}^{\top} \boldsymbol{p} \\
\text { subject to } & \left\|\boldsymbol{q}^{I}\right\|-\mu p^{I} \leqslant 0, \quad I \in \mathcal{C}
\end{array}
$$

Besides accounting for the equations of motion and the sliding friction condition, this problem also reproduces the associated kinematics. In summary, the full set of governing equations, which appears as the first-order Karush-Kuhn-Tucker optimality 
conditions associated with the optimization problem, comprise the linear and angular momentum balance Eq. (12), sliding friction conditions:

$\left\|\boldsymbol{q}^{I}\right\|-\mu p^{I} \leqslant 0, \quad I \in \mathcal{C}$,

$\lambda^{I}\left(\left\|\boldsymbol{q}^{I}\right\|-\mu p^{I}\right)=0, \quad I \in \mathcal{C}$,

$\lambda^{I} \geqslant 0, \quad I \in \mathcal{C}$,

with $\mathcal{C}$ being the set of potential contacts, and the kinematics:

$\boldsymbol{N}_{0}^{\top} \Delta \boldsymbol{x}+\mu \boldsymbol{\lambda}-\boldsymbol{g}_{0}=0$

$\widehat{\boldsymbol{N}}_{0}^{\top} \Delta \boldsymbol{X}-\boldsymbol{R}_{0}^{\top} \Delta \boldsymbol{\alpha}-\boldsymbol{G} \boldsymbol{\lambda}=0$

where the matrix $\boldsymbol{G}$ contains the gradients of $\left\|\boldsymbol{q}^{I}\right\|$. These conditions are again completely analogous to those derived and discussed in [17]. In particular, and with reference to Fig. 1, the first condition limits the relative normal displacement as $\left(\boldsymbol{n}_{0}^{I}\right)^{\top}\left(\Delta \boldsymbol{x}^{i}-\Delta \boldsymbol{x}^{j}\right) \leqslant g_{0}^{I}$ while the second imposes a relation between rotations and tangential displacements. As in the two-dimensional case, the kinematics associated with the variational formulation (13) implies a finite amount of dilation for $\mu>0$. However, as discussed in detail in [17], this dilation may be viewed as an artifact of the time discretization and can be reduced or eliminated entirely by reducing the magnitude of the time step.

\subsection{Alternative $3 D$ formulation}

The three-dimensional granular contact dynamics formulation presented above is in many ways the natural extension of the two-dimensional formulation presented in [17]. The main difference is that the directions of the shear forces are arbitrary subject to the condition that $\left\{\boldsymbol{n}_{0}^{I}, \boldsymbol{a}_{0}^{I}, \boldsymbol{b}_{0}^{I}\right\}$ is an orthonormal basis. In the following, an alternative formulation that does not require such a basis to be established a priori is briefly discussed.

The alternative formulation, sketched in Fig. 2, makes use of a normal force directed along the particle normal as in the previous formulation. The shear forces are accounted for by a separate force vector $\boldsymbol{q}^{I}=\left(q_{x}^{I}, q_{y}^{I}, q_{z}^{I}\right)$ which must satisfy

$\left(\boldsymbol{n}_{0}^{I}\right)^{\top} \boldsymbol{q}^{I}=0$

With these forces, linear momentum balance is given by

$\bar{m}^{i} \Delta \boldsymbol{x}^{i}+p^{I} \boldsymbol{n}_{0}^{I}+\boldsymbol{q}^{I}=\overline{\boldsymbol{f}}_{0}^{i}$

$\bar{m}^{j} \Delta \boldsymbol{x}^{j}-p^{I} \boldsymbol{n}_{0}^{I}-\boldsymbol{q}^{I}=\overline{\boldsymbol{f}}_{0}^{j}$

while the angular momentum balance equations read:

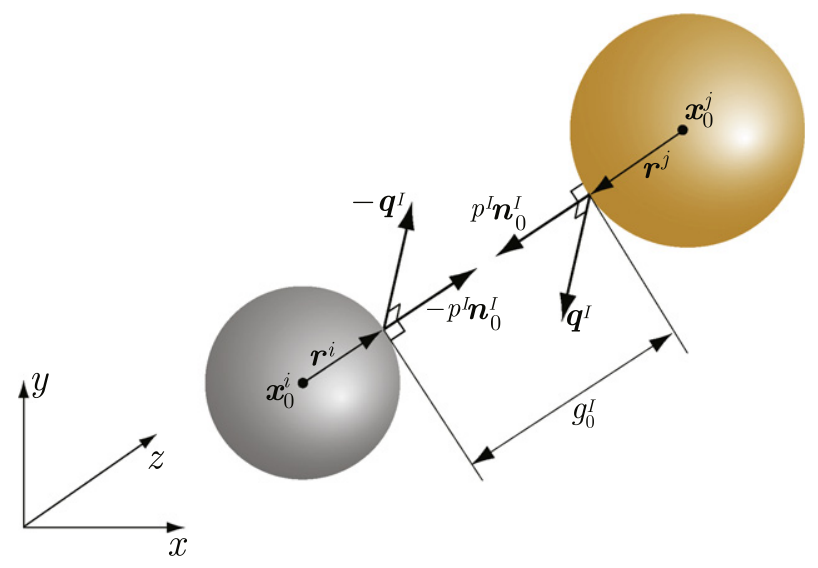

Fig. 2. Alternative 3D formulation: frictional contact geometry.

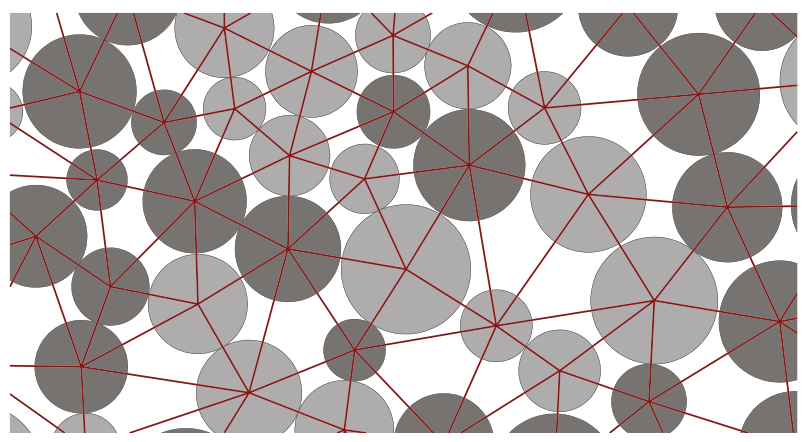

Fig. 3. Potential contacts as given by Delaunay triangulation.

$\bar{J}^{i} \Delta \boldsymbol{\alpha}^{i}-\boldsymbol{r}_{0}^{i} \times \boldsymbol{q}^{I}=\overline{\boldsymbol{m}}_{0}^{i}$

$\bar{J}^{j} \Delta \boldsymbol{\alpha}^{j}+\boldsymbol{r}_{0}^{j} \times \boldsymbol{q}^{I}=\overline{\boldsymbol{m}}_{0}^{j}$

Both these sets of equations are somewhat simpler than those of the previous formulation. As in the previous formulation, the frictional sliding condition is given by

$\left\|\boldsymbol{q}^{I}\right\|-\mu p^{I} \leqslant 0$

In summary, the variational formulation of the alternative $3 \mathrm{D}$ formulation differs from that of (13) by the matrix $\widehat{\boldsymbol{N}}_{0}$ being somewhat simpler and by the additional orthogonality constraint (16). The orthogonality constraint gives rise to an additional set of kinematic variables which makes the kinematics rather less transparent than that associated with the original form of (13). On the other hand, the main quantities of interest, namely the displacements, $\Delta \boldsymbol{x}$ and the rotations, $\Delta \boldsymbol{\alpha}$, are clearly identified.

\subsection{Potential contact specification}

Following [17], potential contacts at $t_{0}+\Delta t$ are defined by a Delaunay triangulation on the basis of the positions at $t_{0}$. This approach is valid in both two and three dimensions. An example of the former is shown in Fig. 3.

\section{Conic programming formulations}

In both the formulations discussed in the previous section, the sliding friction condition is in the form of a second-order cone constraint. Problems involving such constraints can be treated efficiently by means of dedicated methods and a number of very efficient and robust general purpose algorithms are readily available $[17,1,49]$. More generally, conic programming provides a natural and convenient framework for deriving and analyzing variational formulations, for example with respect to duality properties. In the following, these features are utilized for the granular contact dynamics problem.

\subsection{Conic programming duality}

Second-order cone programming often makes use of the following primal standard form $[1,5,18]$ :

$$
\begin{array}{ll}
\text { minimize } & \boldsymbol{c}^{\top} \boldsymbol{x} \\
\text { subject to } & \boldsymbol{A x}=\boldsymbol{b} \\
& \boldsymbol{x}_{i} \in \mathcal{K}_{i}, \quad i=1, \ldots, n
\end{array}
$$

where the variable vector is partitioned into $n$ subvectors such that $\boldsymbol{x}=\left(\boldsymbol{x}_{1}, \ldots, \boldsymbol{x}_{n}\right)$ with each subvector containing elements $\boldsymbol{x}_{i}=\left(x_{i, 1},-\right.$ $\left.\ldots, x_{i, m}\right)$. Each subvector of variables is subject to a conic constraint defined by the cones $\mathcal{K}_{i}$. Of the various possible cones (please refer 
to [5] for an exact definition), the second-order cone is one of the most common. This is given by

$\mathcal{K}: x_{1} \geqslant \sqrt{x_{i, 2}^{2}+\cdots x_{i, m}^{2}}, \quad x_{i, 1} \geqslant 0$

The dual to (20) is given by

$$
\begin{array}{ll}
\text { maximize } & \boldsymbol{b}^{\top} \boldsymbol{y} \\
\text { subject to } & \boldsymbol{A}^{\top} \boldsymbol{y}+\boldsymbol{s}=\boldsymbol{c} \\
& \boldsymbol{s}_{i} \in \mathcal{K}_{i}, \quad i=1, \ldots, n
\end{array}
$$

The gap between the primal and dual solutions is given by

$\boldsymbol{c}^{\top} \boldsymbol{x}-\boldsymbol{b}^{\top} \boldsymbol{y}=\left(\boldsymbol{A}^{\top}+\boldsymbol{s}\right)^{\top} \boldsymbol{x}-(\boldsymbol{A} \boldsymbol{x})^{\top} \boldsymbol{y}=\boldsymbol{s}^{\top} \boldsymbol{x} \geqslant 0$

where the last inequality follows from the definition of the secondorder cone together with the Cauchy-Schwartz inequality.

Consider now the following problem:

$$
\begin{array}{ll}
\text { maximize } & \boldsymbol{w}^{\top} \boldsymbol{\sigma} \\
\text { subject to } & \boldsymbol{B}^{\top} \boldsymbol{\sigma}=\boldsymbol{f} \\
& \boldsymbol{\sigma}_{i} \in \mathcal{K}_{\mu}, \quad i=1, \ldots, n
\end{array}
$$

where the variable vector comprises subvectors $\boldsymbol{\sigma}_{i}=(p, \boldsymbol{q})_{i}$ and the cones are of the Coulomb type:

$\mathcal{K}_{\mu}[p, \boldsymbol{q}]: \mu p \geqslant\|\boldsymbol{q}\|, \quad p \geqslant 0$

The dual to (24) is given by

$$
\begin{array}{ll}
\text { minimize } & -\boldsymbol{f}^{\top} \boldsymbol{u} \\
\text { subject to } & \boldsymbol{B u}-\boldsymbol{e}=-\boldsymbol{w} \\
& \boldsymbol{e}_{i} \in \mathcal{K}_{\mu}^{*}, \quad i=1, \ldots, n
\end{array}
$$

where $\boldsymbol{e}_{i}=(\varepsilon, \gamma)_{i}$ and the dual cones are given by (see Fig. 4):

$\mathcal{K}_{\mu}^{*}[\varepsilon, \boldsymbol{\gamma}]:-\frac{\varepsilon}{\mu} \geqslant\|\boldsymbol{\gamma}\|, \quad \varepsilon \leqslant 0$

Similar to the more conventional standard forms, the duality gap can be shown to be $-\boldsymbol{\sigma}^{\top} \boldsymbol{e} \geqslant 0$.

\subsection{Force based problem}

As shown in [17], the min-max problem (13) is equivalent to the following maximization problem involving only static variables.

$$
\begin{array}{cl}
\text { maximize } & -\frac{1}{2} \boldsymbol{t}^{\top} \overline{\boldsymbol{M}}^{-1} \boldsymbol{t}-\frac{1}{2} \boldsymbol{r}^{\top} \overline{\boldsymbol{J}}^{-1} \boldsymbol{r}-\mathbf{g}_{0}^{\top} \boldsymbol{p} \\
\text { subject to } & \boldsymbol{t}+\boldsymbol{N}_{0} \boldsymbol{p}+\widehat{\boldsymbol{N}}_{0} \boldsymbol{q}=\overline{\boldsymbol{f}}_{0} \\
& \boldsymbol{r}-\boldsymbol{R}_{0} \boldsymbol{q}=\overline{\boldsymbol{m}}_{0} \\
& \left(p^{I}, \boldsymbol{q}^{I}\right) \in \mathcal{K}_{\mu}, \quad I \in \mathcal{C}
\end{array}
$$

where the new variables $\boldsymbol{t}$ and $\boldsymbol{r}$ are to be interpreted as dynamic forces and the Coulomb criterion is expressed as a conic constraint.

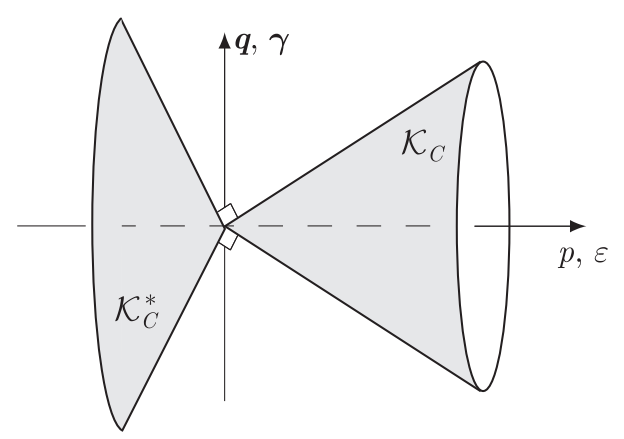

Fig. 4. Primal and dual Coulomb cones.
As discussed in [17], dynamic effects may be omitted from this problem to arrive at the following static problem:

$$
\begin{array}{ll}
\text { maximize } & -\mathbf{g}_{0}^{\top} \boldsymbol{p} \\
\text { subject to } & \boldsymbol{N}_{0} \boldsymbol{p}+\widehat{\boldsymbol{N}}_{0} \boldsymbol{q}=\boldsymbol{f}_{\mathrm{ext}} \\
& \boldsymbol{R}_{0} \boldsymbol{q}=\mathbf{0} \\
& \left(p^{I}, \boldsymbol{q}^{I}\right) \in \mathcal{K}_{\mu}, \quad I \in \mathcal{C}
\end{array}
$$

This problem is similar in structure to those that arise in limit analysis from stress-based formulations of the extremum theorems of plasticity $[14,18,19,27,59,6]$.

\subsection{Displacement based problem}

Similarly, it is possible to derive a problem involving only kinematic variables, i.e. displacements and rotations. The fully dynamic version of this problem can be constructed as follows. First construct the formal dual of the static problem (29). This is given by

$$
\begin{array}{cl}
\text { minimize } & -\boldsymbol{f}_{\mathrm{ext}}^{\top} \Delta \boldsymbol{x} \\
\text { subject to } & \Delta \boldsymbol{u}_{N}=\boldsymbol{N}_{0}^{\top} \Delta \boldsymbol{x} \\
& \Delta \boldsymbol{u}_{T}=\widehat{\boldsymbol{N}}_{0}^{\top} \Delta \boldsymbol{x}-\boldsymbol{R}_{0}^{\top} \Delta \boldsymbol{\alpha} \\
& \left(\Delta u_{N}^{I}-g_{0}^{I}, \Delta \boldsymbol{u}_{T}^{I}\right) \in \mathcal{K}_{\mu}^{*}, \quad I \in \mathcal{C}
\end{array}
$$

Next, dynamic terms expressed in kinematic variables are added to yield:

$$
\begin{array}{ll}
\text { minimize } & \frac{1}{2} \Delta \boldsymbol{x}^{\top} \overline{\boldsymbol{M}} \Delta \boldsymbol{x}-\overline{\boldsymbol{f}}_{0}^{\top} \Delta \boldsymbol{x}+\frac{1}{2} \Delta \boldsymbol{\alpha}^{\top} \overline{\boldsymbol{J}} \Delta \boldsymbol{\alpha}-\overline{\boldsymbol{m}}_{0}^{\top} \Delta \boldsymbol{\alpha} \\
\text { subject to } & \Delta \boldsymbol{u}_{N}=\boldsymbol{N}_{0}^{\top} \Delta \boldsymbol{x} \\
& \Delta \boldsymbol{u}_{T}=\widehat{\boldsymbol{N}}_{0}^{\top} \Delta \boldsymbol{x}-\boldsymbol{R}_{0}^{\top} \Delta \boldsymbol{\alpha} \\
& \left(\Delta u_{N}^{I}-g_{0}^{I}, \Delta \boldsymbol{u}_{T}^{I}\right) \in \mathcal{K}_{\mu}^{*}, \quad I \in \mathcal{C}
\end{array}
$$

Again, it is worth noting that the quasi-static problem (30) is similar in structure to common kinematic formulations of limit analysis $[35,56,3,54,55]$. In analogy with the force-based yield condition in (29), the relative normal and tangential displacement are now limited by a conic constraint which effectively imposes the flow rule.

\subsection{Implementation}

In [17], the force based problem (28) was chosen as the problem actually solved by the general purpose solver SONIC [16]. However, it appears that the kinematic equivalent (31) is somewhat more favorable as no additional variables need to be introduced. In practice, however, the difference in solution time between the two problems is relatively minor, but with (31) definitely being preferable.

\section{Rolling resistance}

The particles that make up most natural granular materials are far from spherical and their idealization in terms of spheres may lead to quite erroneous results. In particular, it has long been recognized that the resistance to rolling of non-spherical particles plays a major role in a wide range of problems, from highly dynamic granular flows to common quasi-static soil mechanics laboratory tests $[31,9,36,8,60]$. While the obvious remedy to accounting for particle shape in simulations is to use particles that better represent the actual shape, this is usually only realized at a significantly higher degree of complexity and computational cost.

An attractive alternative is therefore to incorporate some finite amount of rolling resistance into the basic sphere model. This is usually done by modifying the governing equations such that a moment above a certain threshold is required for rolling to 


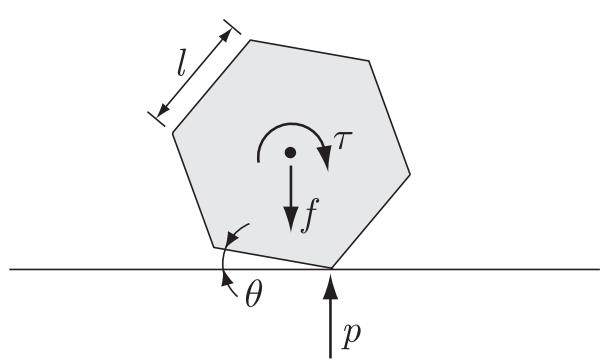

Fig. 5. Rolling resistance of two-dimensional hexagonal particle.

commence. The principle is illustrated in Fig. 5 where a hexagonal particle with a body force $f$ is considered. In order for rolling to commence, i.e. in order for the angle $\theta$ to increase above zero, a moment $\tau=\tau_{\max }=\frac{1}{2} p l$ is required. It is here evident that the rolling resistance is generated by the eccentricity of the contact force $p$. In order to map the hexagon into an equivalent disk, it is necessary to establish a relation between the side length of the hexagon, $l$, and the radius of the disk. This can be done in various ways, the most obvious being to either assume equal perimeters or equal areas. The first approach leads to $l=\frac{1}{3} \pi r=1.047$ while the second implies $l=1.010 r$. In addition, the moment necessary for rolling to commence is not representative of the average moment required during the course of a full revolution. Indeed, in the present case, the resisting moment attains its maximum value for $\theta=0^{\circ}$ and then decreases to become equal to zero at $\theta=60^{\circ}$ and remains zero until $\theta=120^{\circ}$. This suggests an average resisting moment of $\tau=\frac{1}{4} \tau_{\max }$. Using the above mentioned relation between side length and radius, we have a rolling resistance of

$|\tau|=\frac{1}{8} p l=\mu_{r} r p$

where $\mu_{r}$ is referred to as the rolling friction coefficient in analogy with the sliding friction coefficient. Assuming identical perimeters for the hexagon and the disk, we have $\mu_{r}=0.131$ while assuming identical areas gives $\mu_{r}=0.137$. A more refined analysis along similar lines has recently been carried out by Estrada et al. [9] who, for a general regular polygon with $n_{s}$ sides, found

$\mu_{r}=\frac{1}{2} \tan \left(\frac{\pi}{2 n_{s}}\right)$

The approach taken to arrive at this expression was to match the total work required to rotate the polygon and an equivalent disk through a full cycle using the equal perimeter approach to define the equivalent radius. For $n_{s}=6$, this gives $\mu_{r}=0.134$, in good agreement with the result of the simple analysis presented above.

\subsection{Rolling resistance in $3 D$}

In the three-dimensional case, we may proceed by using the same decomposition of the three moment components $\tau=\left(\tau_{x}, \tau_{y}\right.$,$\tau_{z}$ ) as for the contact forces:

$\tau^{I}=\boldsymbol{n}_{0}^{I} \tau_{N}^{I}+\tau_{T}^{I}$

where $\tau_{T}^{I}=\boldsymbol{a}_{0}^{I} \tau_{T \alpha}^{I}+\boldsymbol{b}_{0}^{I} \tau_{T \beta}^{I}$. Besides the fact that three moment components are now present, an important point that distinguishes the two-and three-dimensional cases is that the physical origins of the normal, or torsional, moment $\tau_{N}$ is quite different from that of the two tangential moments, $\tau_{\alpha}$ and $\tau_{\beta}$. Indeed, while the two latter are generated by contact force eccentricities and hence depend on the particle shape alone, the torsional moment depends on the geometry of the contact surface, the normal contact force, and the sliding friction coefficient of the contact. For the simple case of a cubic particle with side lengths $l$ on a plane surface, the maximum torsional moment would be $\left|\tau_{\max }\right|=\mu l p$. In the more general case, we may relate the torsional moment to the normal force by the inequality

$\left|\tau_{N}^{I}\right|-\alpha_{t} \mu r p^{I} \leqslant 0$

where $\alpha_{t}$ is a shape factor that should be close to 1 for a cubic specimen (depending on how side length is related to sphere radius) and gradually decrease to zero for a perfectly spherical particle.

The tangential moments, which are generated by contact force eccentricities can, as a direct extension of the two-dimensional relation, be related to the normal force by

$\left\|\tau_{T}^{I}\right\|-\mu_{r} r p^{I} \leqslant 0$

This relation implicitly assumes that the particles are isotropic in the sense that, on average, no direction of rolling is preferred over others.

It should be noted that the above rolling resistance model only accounts for general non-spherical particles in an average, or effective, manner. As such, for natural grains which often are quite irregular, the value of $\mu_{r}$ must be determined by a process of calibration. The two-dimensional results discussed above are here useful as a means of providing an initial estimate. The extreme limits of $\mu_{r}$ are 0 (corresponding to a disk) and 0.21 (corresponding to a square), so for many natural materials $\mu_{r}=0.1$ (corresponding to an octagon) provides a reasonable initial estimate.

\subsection{Particle-to-particle contact}

In the above, we have only considered the rolling resistance encountered in the contact between a particle and a plane surface. The case of particle-to-particle contact can be handled in the following way. Firstly, following the treatment of inter-particle contact forces, we consider a set of self-equilibrated moments at each contact. The angular momentum balance equations in (1) are then extended as

$\boldsymbol{r}-\boldsymbol{R}_{0} \boldsymbol{q}=\overline{\boldsymbol{m}}_{0}+\boldsymbol{N}_{0} \tau_{N}+\widehat{\boldsymbol{N}}_{0} \tau_{T}$

Secondly, when enforcing the rolling resistance conditions (35) and (36) for a contact involving particles with different radii, a decision must be made regarding the radius, $r$, used in these conditions. A common choice, e.g. [31], is to define the common radius as the geometric mean of the radii of the contacting particles. However, bearing in mind that the resisting moment essentially is generated by a contact force eccentricity, it would seem more reasonable to take the common radius as the minimum radius of the contacting particles. In this way, the resistance generated by a plane surface (a particle of infinite radius) is the same as that generated by the contact between two particles of the same radius, in agreement with the premises of the analysis in Section 4.2. Therefore, in the following, the common radius used to define the rolling resistance generated at a contact between two particles of different radius is taken as the radius of the smaller particle.

\subsection{Force based problem}

A force based problem incorporating rolling resistance may now be constructed by substituting the augmented angular momentum balance Eq. (37) into (28) and adding the rolling resistance conditions (35) and (36). Furthermore, for the sake of brevity and since it is of negligible effect in typical simulations, we assume that the torsional resistance is zero. We then have 


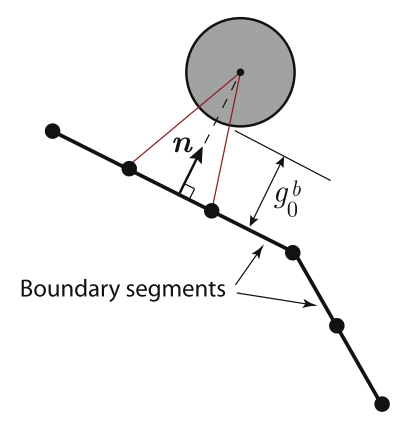

(a)

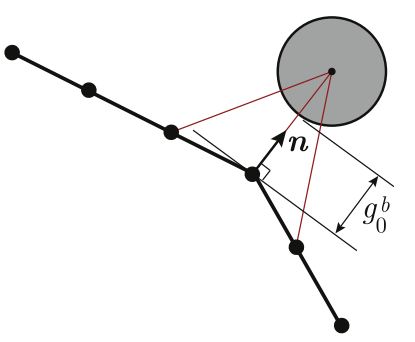

(b)
Fig. 6. Potential boundary contact specification.

$$
\begin{array}{cl}
\text { maximize } & -\frac{1}{2} \boldsymbol{t}^{\top} \overline{\boldsymbol{M}}^{-1} \boldsymbol{t}-\frac{1}{2} \boldsymbol{r}^{\top} \overline{\boldsymbol{J}}^{-1} \boldsymbol{r}-\mathbf{g}_{0}^{\top} \boldsymbol{p} \\
\text { subject to } & \boldsymbol{t}+\boldsymbol{N}_{0} \boldsymbol{p}+\widehat{\boldsymbol{N}}_{0} \boldsymbol{q}=\overline{\boldsymbol{f}}_{0} \\
& \boldsymbol{r}-\boldsymbol{R}_{0} \boldsymbol{q}=\overline{\boldsymbol{m}}_{0}+\widehat{\boldsymbol{N}}_{0} \boldsymbol{\tau}_{T} \\
& \left(p^{I}, \boldsymbol{q}^{I}\right) \in \mathcal{K}_{\mu}, \quad I \in \mathcal{C} \\
& \left(r p^{I}, \tau_{T}^{I}\right) \in \mathcal{K}_{\mu_{r}}, \quad I \in \mathcal{C}
\end{array}
$$

The constraints here form a quarter four-sided cone in $\left(p,\|\boldsymbol{q}\|,\left\|\boldsymbol{\tau}_{T}\right\|\right)$ space.

\subsection{Displacement based problem}

Following the procedure of Section 3, the displacement based equivalent of the force based problem (38) is given by

$$
\begin{array}{cl}
\text { minimize } & \frac{1}{2} \Delta \boldsymbol{x}^{\top} \overline{\boldsymbol{M}} \Delta \boldsymbol{x}-\overline{\boldsymbol{f}}_{0}^{\top} \Delta \boldsymbol{x}+\frac{1}{2} \Delta \boldsymbol{\alpha}^{\top} \overline{\boldsymbol{J}} \Delta \boldsymbol{\alpha}-\overline{\boldsymbol{m}}_{0}^{\top} \Delta \boldsymbol{\alpha} \\
\text { subject to } & \Delta \boldsymbol{u}_{N}=\boldsymbol{N}_{0}^{\top} \Delta \boldsymbol{x} \\
& \Delta \boldsymbol{u}_{T}=\widehat{\boldsymbol{N}}_{0}^{\top} \Delta \boldsymbol{x}-\boldsymbol{R}_{0}^{\top} \Delta \boldsymbol{\alpha} \\
& \Delta \boldsymbol{\omega}_{N}=-\boldsymbol{N}_{0}^{\top} \Delta \boldsymbol{\alpha} \\
& \Delta \boldsymbol{\omega}_{T}=-\widehat{\boldsymbol{N}}_{0}^{\top} \Delta \boldsymbol{\alpha} \\
& \left(\Delta u_{N}^{I}-g_{0}^{I}, \Delta \boldsymbol{u}_{T}^{I}\right) \in \mathcal{K}_{\mu}^{*}, \quad I \in \mathcal{C} \\
& \left(\frac{1}{r} \Delta \omega_{N}^{I}, \Delta \boldsymbol{\omega}_{T}^{I}\right) \in \mathcal{K}_{\mu_{r}}^{*}, \quad I \in \mathcal{C}
\end{array}
$$

where $\omega_{N}$ and $\omega_{T}$ are the normal and tangential rotations. Again the constraints are coupled, this time through the global rotations $\alpha$.

\section{Boundary conditions}

Boundary conditions may be included in the final optimization problems (38) or (39) to account for rigid walls with or without prescribed forces. These types of boundary conditions are covered in the following along with a general methodology for specifying potential boundary contacts given a collection of boundary segments (in 2D) or planes (in 3D).

\subsection{Potential boundary contact specification}

The basic approach to specifying boundary constraints follows that of general particle-to-particle contact closely. Consider the two-dimensional case and suppose that the two segments shown in Fig. 6a constitute a rigid boundary. With the end points of each segment given, a number of additional points are inserted between the end points. When performing the Delaunay triangulation at the beginning of each time step, these additional boundary points as well as the end points are included. In this way, potential contacts between the particles and the boundary points are established. Next, for each particle that has a potential contact to a boundary segment, a projection onto the segment is considered. If the resulting point falls between the end point, the closest point has been determined and the relevant non-penetration constraint is given by

$\boldsymbol{n}^{\top} \Delta \boldsymbol{x}^{I} \leqslant g_{0}^{b}$

where $g_{0}^{b}$ is the gap between the particle and the boundary segment, i.e. the minimum distance, and $\boldsymbol{n}$ is its normal.

On the other hand, if the projection onto the boundary segment falls outside the segment, the closest end point is taken as the contact point. This situation is shown in Fig. $6 \mathrm{~b}$ and allows for a straightforward treatment of both convex and non-convex boundaries.

In three dimensions a similar procedure can be used. Consider the case where the boundary is discretized by triangles. The particle center is first projected onto the triangle. If the resulting point is not contained within the triangle, three separate projections are performed with respect to each of the segments defining the triangle. The resulting distances are then compared with the distances to the triangle vertices and the point among these that minimizes the distance is taken as the contact point. We note that significant improvements can be made with this somewhat brute force approach, though, in practice, the identification of potential boundary supports constitutes an insignificant part of the total computational time.

\subsection{Rigid frictional boundaries}

With the identification of the potential boundary contacts, the inclusion of rigid frictional boundary constraints into the final optimization is straightforward. In the force based problems (28) and (38) new sets of boundary contact forces $\boldsymbol{p}^{b}$ and $\boldsymbol{q}^{b}$ are introduced in the linear momentum balance equations which then read:

$\boldsymbol{t}+\boldsymbol{N}_{0} \boldsymbol{p}+\widehat{\boldsymbol{N}}_{0} \boldsymbol{q}+\boldsymbol{N}_{0}^{b} \boldsymbol{p}^{b}+\widehat{\boldsymbol{N}}_{0}^{b} \boldsymbol{q}^{b}=\overline{\boldsymbol{f}}_{0}$

where $\boldsymbol{N}_{0}^{b}$ and $\widehat{\boldsymbol{N}}_{0}^{b}$ contain the boundary unit vectors. Similarly, the angular momentum balance is extended to include the contribution from boundary shear forces:

$\boldsymbol{r}-\boldsymbol{R}_{0} \boldsymbol{q}-\boldsymbol{R}_{0}^{b} \boldsymbol{q}^{b}=\overline{\boldsymbol{m}}_{0}+\widehat{\boldsymbol{N}}_{0} \boldsymbol{\tau}_{T}+\widehat{\boldsymbol{N}}_{0}^{b} \tau_{T}^{b}$

where boundary moments $\tau_{T}^{b}$ have been introduced and $\boldsymbol{R}_{0}^{b}$ is similar to $\boldsymbol{R}_{0}$ but contains only contributions from boundary particles. Furthermore, frictional sliding and rolling resistance conditions equivalent to those imposed for particle-to-particle contact are included. Finally, the objective function is amended by the term $\left(\mathbf{g}_{0}^{b}\right)^{\top} \boldsymbol{p}^{b}$, where $\mathbf{g}_{0}^{b}$ contains the boundary gaps.

\subsection{Resultant force constraints}

Often it is useful to impose constraints that limit the maximum resultant force on a rigid boundary. Such constraints are particularly relevant in common soil mechanics laboratory tests such as biaxial or triaxial tests. Such constraints are readily included into the forced based problems, simply by imposing a maximum magnitude of the resultant contact forces. Thus, the quasi-static force based problem (29) would be of the type:

$$
\begin{array}{cl}
\text { maximize } & -\boldsymbol{g}_{0}^{\top} \boldsymbol{p}-\left(\boldsymbol{g}_{0}^{b}\right)^{\top} \boldsymbol{p}^{b} \\
\text { subject to } & \boldsymbol{N}_{0} \boldsymbol{p}+\widehat{\boldsymbol{N}}_{0} \boldsymbol{q}+\boldsymbol{N}_{0}^{b} \boldsymbol{p}^{b}+\widehat{\boldsymbol{N}}_{0}^{b} \boldsymbol{q}^{b}=\boldsymbol{f}_{\text {ext }} \\
& \boldsymbol{R}_{0} \boldsymbol{q}+\boldsymbol{R}_{0}^{b} \boldsymbol{q}^{b}=\mathbf{0} \\
& \boldsymbol{A} \boldsymbol{p}^{b} \leqslant \boldsymbol{f}_{b} \\
& \left(p^{I}, \boldsymbol{q}^{I}\right) \in \mathcal{K}_{\mu}, \quad I \in \mathcal{C} \\
& \left(p^{I}, \boldsymbol{q}^{I}\right)^{b} \in \mathcal{K}_{\mu}, \quad I \in \mathcal{C}^{b}
\end{array}
$$

where $\boldsymbol{f}_{b}$ are the limiting resultant forces. The Lagrange multipliers associated with the additional equality constraints may be 
interpreted as the displacements of the boundaries. Indeed, the dual to the above problem is the displacement-based problem:

$$
\begin{array}{ll}
\text { minimize } & -\boldsymbol{f}_{\text {ext }}^{\top} \Delta \boldsymbol{x}-\boldsymbol{f}_{b}^{\top} \Delta \boldsymbol{x}^{b} \\
\text { subject to } & \Delta \boldsymbol{u}_{N}=\boldsymbol{N}_{0}^{\top} \Delta \boldsymbol{x} \\
& \Delta \boldsymbol{u}_{T}=\widehat{\boldsymbol{N}}_{0}^{\top} \Delta \boldsymbol{x}-\boldsymbol{R}_{0}^{\top} \Delta \boldsymbol{\alpha} \\
& \Delta \boldsymbol{u}_{N}^{b}=\left(\boldsymbol{N}_{0}^{b}\right)^{\top} \Delta \boldsymbol{x} \\
& \Delta \boldsymbol{u}_{T}^{b}=\left(\widehat{\boldsymbol{N}}_{0}^{b}\right)^{\top} \Delta \boldsymbol{x}-\left(\boldsymbol{R}_{0}^{b}\right)^{\top} \Delta \boldsymbol{\alpha} \\
& \left(\Delta u_{N}^{I}-g_{0}^{I}, \Delta \boldsymbol{u}_{T}^{I}\right) \in \mathcal{K}_{\mu}^{*}, \quad I \in \mathcal{C} \\
& \left(\Delta u_{N}^{I}-g_{0}^{I}, \Delta \boldsymbol{u}_{T}^{I}\right)^{b} \in \mathcal{K}_{\mu}^{*}, \quad I \in \mathcal{C}^{b}
\end{array}
$$

where the new variables, $\Delta \boldsymbol{x}^{b}$, associated with the additional equality constraints, are the displacements of the boundaries.

\section{Examples}

In the following, two examples demonstrating the capabilities of the proposed method are presented with the influence of rolling resistance being particularly emphasized. All optimization problems generated by the granular contact dynamics formulation have been solved using the code SONIC which has previously been developed with continuum plasticity applications in mind [16], though it is also well suited for the present application.

\subsection{True triaxial test}

The first example concerns the simulation of a true triaxial test and makes use of the quasi-static version of the scheme. The sample, shown in Fig. 7, comprises approximately 8000 massless particles. The particle size distribution is uniform with a $\mathrm{min} / \mathrm{max}$ diameter ratio of $d_{\min } / d_{\max }=0.5$ and an initial porosity of 0.38 , cor-

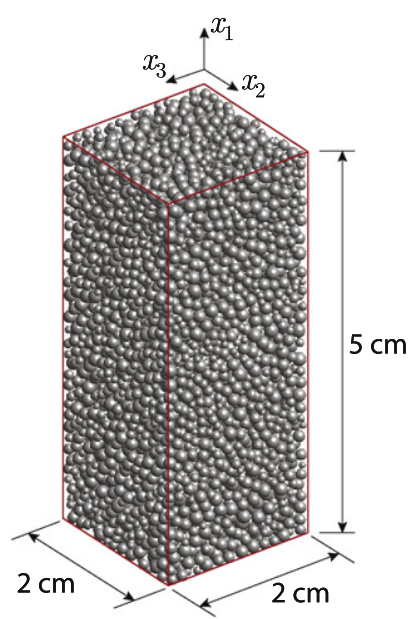

Fig. 7. True triaxial test setup.

responding to a relatively loose packing. The sliding friction coefficient and rolling resistance coefficient were set to zero during the generation of the assembly which was realized by depositing particles into a rectangular box as indicated in Fig. 7. After the preparation of the sample, the particle-to-particle friction coefficient was set to $\mu=\tan 30^{\circ}=0.577$ in all tests while the rolling friction coefficient was varied between tests. The triaxial test was modeled by confining the packing between six smooth walls. The top and bottom boundaries were allowed to move vertically as loading platens. With the mean stress maintained constant at

$\sigma_{1}+\sigma_{2}+\sigma_{3}=300 \mathrm{kPa}$

the specimen was subjected to radial deviatoric loading paths in which the ratio
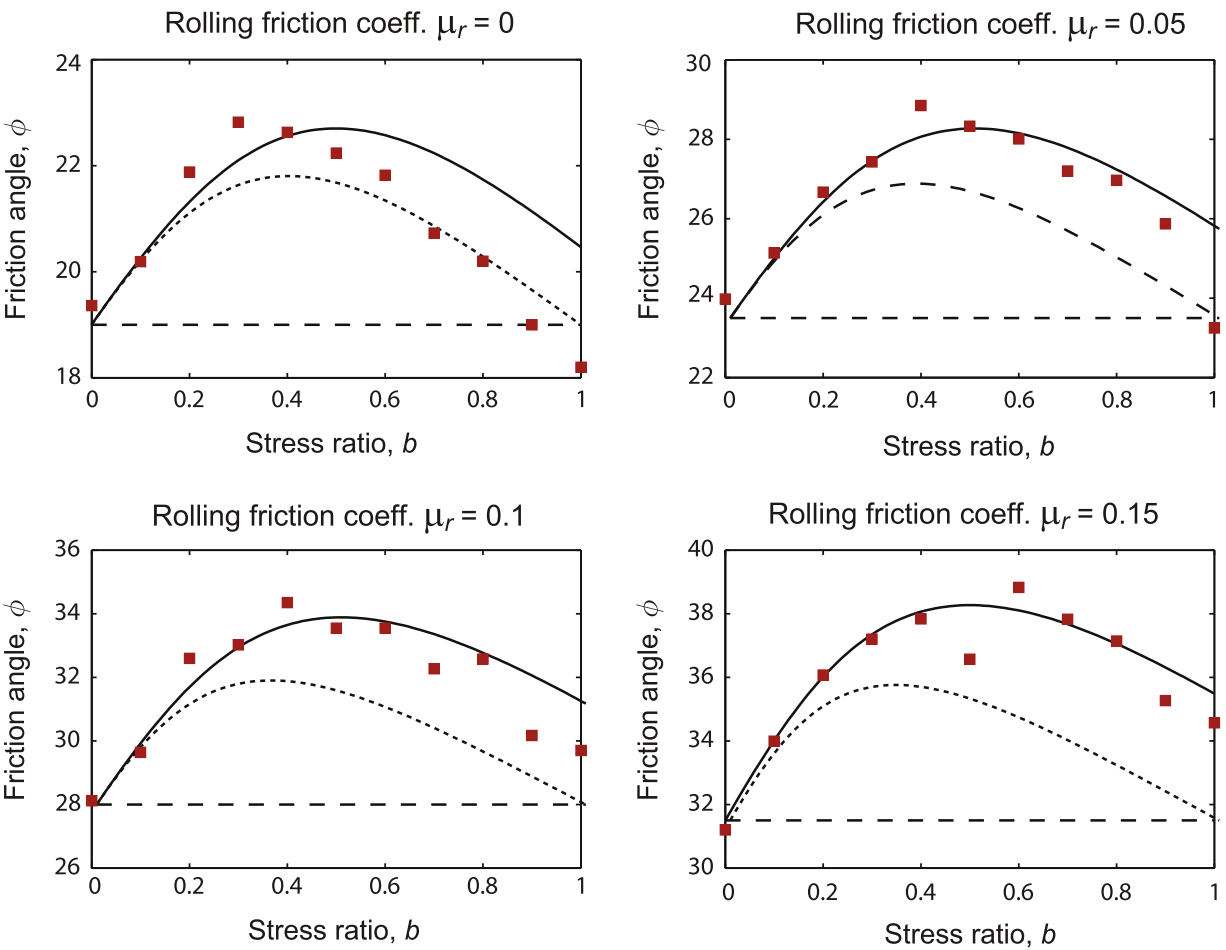

Lade-Duncan _.....- Matsuoka-Nakai _ - - Mohr-Coulomb @ GCD

Fig. 8. Comparison of granular contact dynamics results with common failure criteria. 


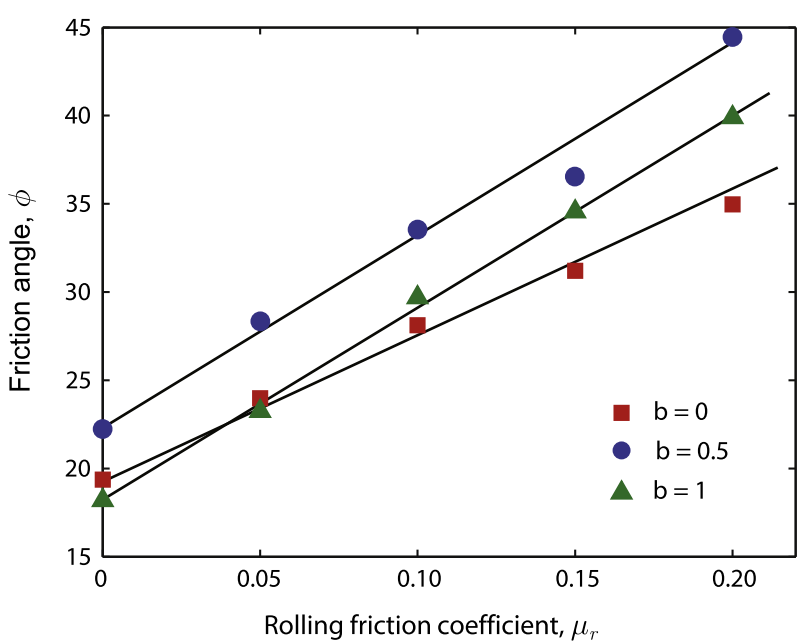

Fig. 9. True triaxial test: dependence of macroscopic friction angle on rolling friction coefficient.

$b=\frac{\sigma_{2}-\sigma_{3}}{\sigma_{1}-\sigma_{3}}$

was kept constant in each test. In this way the deviatoric stress is monotonically increased along different radial stress paths on the $\pi$-plane of principal stress space. The conventional axisymmetric compression test thus corresponds to $b=0$, while triaxial extension corresponds to $b=1$. A total of 500 time steps of equal magnitude were used to impose a total axial strain of 0.5 . For each radial loading test simulation, the state of stress corresponding to failure was identified and the friction angle, $\phi$, defined by

$\sin \phi=\frac{\sigma_{1}-\sigma_{3}}{\sigma_{1}+\sigma_{3}}$

was recorded. The results, in terms of friction angle as function of $b$ and for different rolling friction coefficients, are shown in Fig. 8. Also shown in this figure are traces of the Lade-Duncan [22], Matsuoka-Nakai [29] and Mohr-Coulomb criteria, matched for triaxial compression and fitted to the former criterion. As seen, the agreement with the Lade-Duncan criterion is rather good, especially for higher values of $\mu_{r}$. In particular, the results verify the different triaxial compression and extension friction angles predicted by this criterion. Similar findings have previously been made by Thornton [53] using the conventional DEM.

Regarding rolling resistance, we note that increasing $\mu_{r}$ increases the macroscopic friction angle, for $b=0$ from around $19^{\circ}$ for $\mu_{r}$ to as much as $31.5^{\circ}$ for $\mu_{r}=0.15$. In fact, a more careful analysis shows that the relation between the macroscopic friction angle and the microscopic rolling friction coefficient to a very good approximation is linear (see Fig. 9).

\subsection{Granular column collapse}

The second example involves the collapse of an initially cylindrical column under the action of gravity. Due to its apparent simplicity and a number of somewhat surprising scaling laws, this problem has been the subject of several investigations since first being introduced in a series of experiments by Lube et al. [26,25] and Lajeunesse et al. [24]. Both discrete models of the kind covered in this paper $[21,20,58,45,46,23]$ and continuum models $[28,13,23]$ have been proposed and shown to reproduce many of the features of the physical experiments. So far, however, there have been few attempts to reproduce the experimentally observed behavior quantitatively using these models.
For particle models, the main challenge in reproducing the behavior of natural grains such as those used in the above mentioned experiments is that these inevitably deviate considerably from perfect spheres. To assess the ability of the rolling resistance model to capture the effects of the non-spherical geometries, a series of simulations were conducted using the fully dynamic version of the granular contact dynamics scheme. An example of the setup is shown in Fig. 10. The column is generated by letting particles,

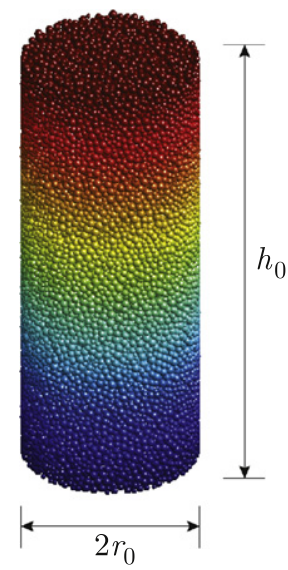

Fig. 10. Granular column with $h_{0} / r_{0}=5$.
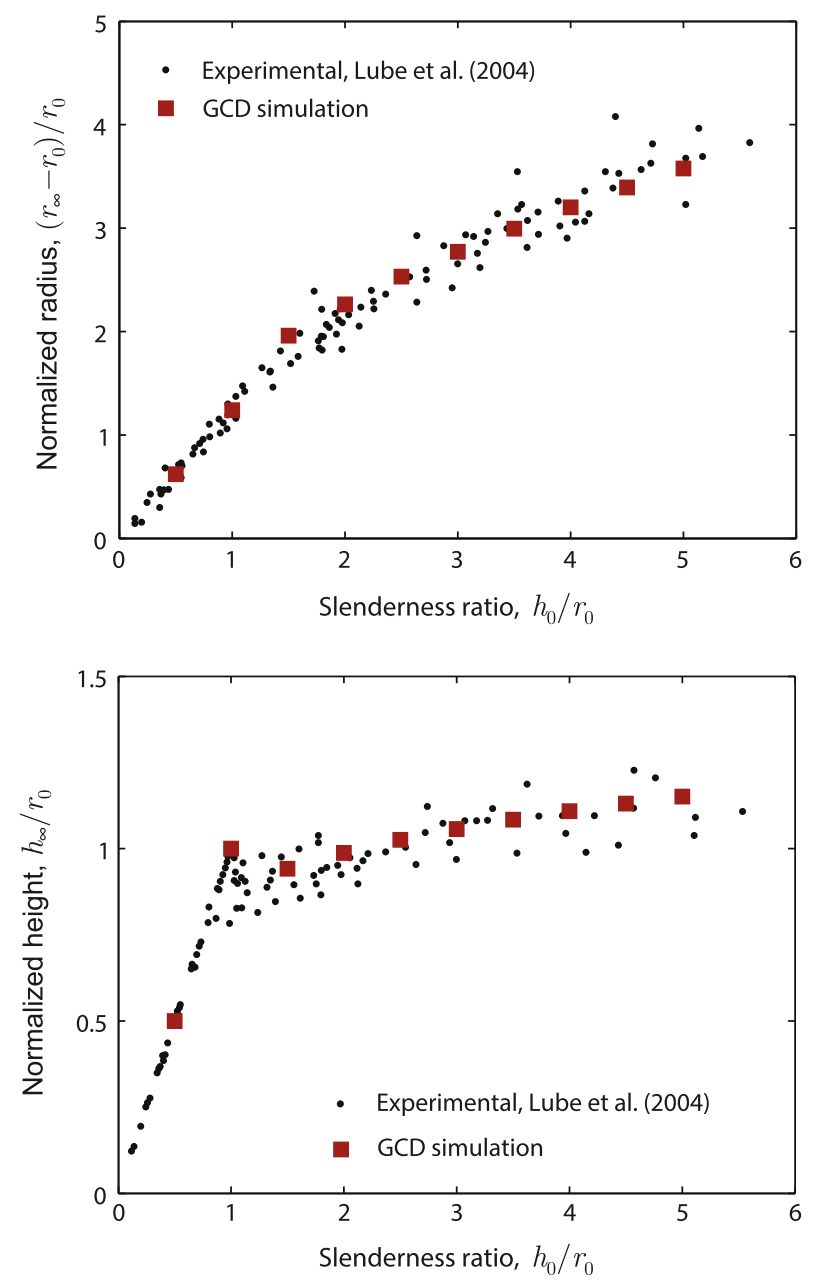

Fig. 11. Granular column collapse: dependence of final radius and height on the slenderness ratio. 


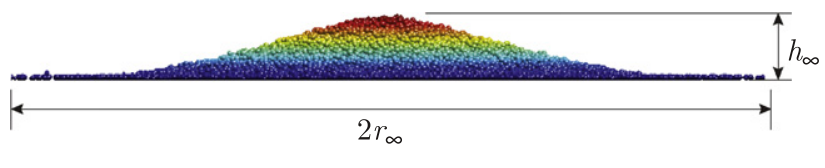

Fig. 12. Granular column collapse: final deposit for column with $h_{0} / r_{0}=5$.

approximately 40,000 , fall freely into a cylinder with a given base radius, $r_{0}$. After the particles have settled, the column is trimmed to a specified height, $h_{0}$. As in the previous example, we use $d_{\min } /$ $d_{\max }=0.5$ and the initial density is controlled by adjusting the inter-particle friction coefficient so that a porosity of 0.42 (corresponding to that used in [26]) is achieved. The column then collapses under the action of gravity, i.e. all boundaries except the bottom one are released. The simulations are carried out for a total time of $\bar{t}=t / \sqrt{h_{0} / g}=4.0$ using 100 time steps of equal magnitude. The inter-particle friction coefficient is set to $\mu=\tan 30^{\circ}=0.577$ while the rolling friction coefficient is set to $\mu_{r}=0.12$ in all simulations. This value was chosen on the basis of the results of the previous example in that it corresponds approximately to a macroscopic friction angle $30^{\circ}$. In two dimensions, the $\mu_{r}=0.12$ corresponds to a shape in between a hexagon $\left(n_{s}=6\right.$, $\left.\mu_{r}=0.13\right)$ and a heptagon $\left(n_{s}=7, \mu_{r}=0.11\right)$.

Under these conditions, a series of simulations for columns with different slenderness ratios, $h_{0} / r_{0}$, were conducted and the final radius and height were recorded. The results are shown in Fig. 11 along with the experimental data of Lube et al. [26]. As seen, the match between experiment and simulation is rather good. In particular, the approximately bilinear dependence of the final radius and height on the slenderness ratio is captured well.

In Fig. 12, the final deposit for a column with $h_{0} / r_{0}=5$ is shown. Compared to the triangular form that would be expected in a static version of the experiment, a more conical shape is observed, again in good agreement with physical experiments.

This example demonstrates that the rolling resistance model presented is capable of capturing the physical behavior of natural, non-spherical, particles quite well. Although a calibration needs to be carried out in order to determine the appropriate value of $\mu_{r}$, this seems to be a small price to pay compared to the alternative of accounting for the actual particle geometries in the simulations.

\section{Conclusions}

The conclusions are as follows:

- Two different three-dimensional formulations of granular contact dynamics as it applies to spherical particles have been developed with the variational structure of the formulations being particularly emphasized (Section 2). Both static and dynamic versions of the formulations have been derived. Notably, the former appears as a special case of the latter and the same algorithms are applicable to both versions of the scheme. This avoids, among other things, the need for approximating quasi-static problems in terms of their fully dynamic counterparts as in the conventional DEM.

- Both formulations can be cast in terms of second-order cone programs. An analysis of the duality properties within the framework of conic programming has been presented (Section 3 ). This allows for a straightforward interpretation both in terms of static and kinematic quantities. While the two problems, static and kinematic, are equivalent, the kinematic problem appears to be favorable in an algorithmic context as the number of variables is somewhat smaller.

- To account for the effects of non-spherical geometries in an 'effective' manner, a rolling resistance model has been proposed (Section 4). This is incorporated into the variational formulation in a straightforward manner and does not require any algorithmic modifications. While the model requires an additional material parameter (in addition to the inter-particle friction coefficient), the magnitude of this can be estimated on the basis of a simple two-dimensional analogy involving arbitrary regular polyhedra. Although there would certainly be cases in which the proposed rolling resistance would fail to provide a reasonable representation of reality, its utility in typical problems has been demonstrated by means of numerical examples and comparisons to physical experiments (Section 6).

- Finally, the crucial issue of imposing boundary conditions has been discussed in some detail (Section 5). The scheme here used follows that of inter-particle contact quite closely and can be used to model, in principle, arbitrarily complex rigid boundaries. Flexible boundaries have not been considered, but appear to be possible to model using a similar approach to enforce contact between particles and flexible finite elements.

\section{References}

[1] Andersen ED, Roos C, Terlaky T. On implementing a primal-dual interior-point method for conic quadratic optimization. Mathe Programm 2003;95:249-77.

[2] Anitescu M, Potra FA. A time-stepping method for stiff multi-rigid-body dynamics with contact and friction. Int J Numer Meth Eng 2002;55:753-84.

[3] Antao AN, da Silva M Vicente, Guerra N, Delgado R. An upper bound-based solution for the shape factors of bearing capacity of footings under drained conditions using a parallelized mixed f.e. formulation with quadratic velocity fields. Comput Geotech 2012;41:23-35.

[4] Bartels G, Unger T, Kadau D, Wolf DE, Kertesz J. The effect of contact torques on porosity of cohesive powders. Granul Matt 2005;7:139-43.

[5] Ben-Tal A, Nemirovski A. Lectures on modern convex optimization: analysis, algorithms, and engineering applications. MPS-SIAM Series on Optimization; 2001.

[6] Borges LA, Zouain N, Huespe AE. A nonlinear optimization procedure for limit analysis. Euro J Mech, A/Solids 1996;15(3)::487-512.

[7] Cundall PA, Strack ODL. A discrete numerical model for granular assemblies. Geotechnique 1979;29:47-65.

[8] Donev A, Cisse I, Sachs D, Variano EA, Stillinger FH, Connelly R, et al. Improving the density of jammed disordered packings using ellipsoids. Science 2004;303(5660):990-3.

[9] Estrada N, Azema E, Radjai F, Taboada A. Identification of rolling resistance as a shape parameter in sheared granular media. Phys Rev E 2011;84:011306.

[10] Jean M. The non-smooth contact dynamics method. Comput Meth Appl Mech Eng 1999;177:235-57.

[11] Kadau D, Jr, A JS, Herrman HJ. A micromechanical model of collapsing quicksand. Granul Matt 2011;13:219-23.

[12] Kadau D, Schwesig D, Theuerkauf J, Wolf DE. Influence of particle elasticity in shear testers. Granul Matt 2006;8:35-40.

[13] Kerswell RR. Dam break with Coulomb friction: a model for granular slumping? Phys Fluids 2005;17:057101.

[14] Krabbenhoft K, Damkilde L. A general nonlinear optimization algorithm for lower bound limit analysis. Int J Numer Meth Eng 2003;56:165-84.

[15] Krabbenhoft K, Huang J, da Silva MV, Lyamin AV. Granular contact dynamics with particle elasticity. Granular Matter 2012;14:607-19.

[16] Krabbenhoft K, Lyamin AV. Computational Cam clay plasticity using secondorder cone programming. Comput Meth Appl Mech Eng 2012;209-212: 239-49.

[17] Krabbenhoft K, Lyamin AV, Huang J, da Silva MV. Granular contact dynamics using mathematical programming methods. Comput Geotech 2012;43: 165-76.

[18] Krabbenhoft K, Lyamin AV, Sloan SW. Formulation and solution of some plasticity problems as conic programs. Int J Solids Struct 2007;44:1533-49.

[19] Krabbenhoft K, Lyamin AV, Sloan SW. Three-dimensional Mohr-Coulomb limit analysis using semidefinite programming. Commun Numer Meth Eng 2008;24:1107-19.

[20] Lacaze L, Kerswell RR. Axisymmetric granular collapse: a transient 3D flow test of viscoplasticity. Phys Rev Lett 2009;102:108305.

[21] Lacaze L, Phillips JC, Kerswell RR. Planar collapse of a granular column: experiments and discrete element simulations. Phys Fluids 2008;20:063302.

[22] Lade PV, Duncan JM. Elastoplastic stress-strain theory for cohesionless soil. J Geotech Eng Div, ASCE 1975;101:1037-53.

[23] Lagree PY, Staron L, Popinet S. The granular column collapse as a continuum: validity of a two-dimensional Navier-Stokes model with a $\mu(\mathrm{i})$-rheology. J Fluid Mech, In press.

[24] Lajeunesse E, Mangeney-Castelneau A, Vilotte JP. Spreading of a granular mass on an horizontal plane. Phys Fluids 2004;16. 2731-381.

[25] Lube G, Sparks HEHRSJ, Freundt A. Collapses of two-dimensional granular columns. Phys Rev E 2005;72:041301. 
[26] Lube G, Sparks HEHRSJ, Hallworth MA. Axisymmetric collapses of granular columns. J Fluid Mech 2004;508:175-99.

[27] Lyamin AV, Sloan SW, Krabbenhoft K, Hjiaj M. Lower bound limit analysis with adaptive remeshing. Int J Numer Meth Eng 2005;63:1961-74.

[28] Mangeney-Castelnau A, Bouchut F, Vilotte JP, Lajeunesse E, Aubertin A, Pirulli M. On the use of saint venant equations to simulate the spreading of a granular mass. J Geophys Res 2005;110:B09103.

[29] Matsuoka H, Nakai T. Stress-deformation and strength characteristics of soil under three different principal stresses. Proc Jpn Soc Civil Eng 1974;232: 59-70.

[30] McNamara S, Herrmann H. Measurement of indeterminacy in packings of perfectly rigid disks. Phys Rev E 2004;70:061303.

[31] Mohamed A, Gutierrez M. Comprehensive study of the effects of rolling resistance on the stress strain and strain localization behavior of granular materials. Granul Matt 2010;12:527-41.

[32] Moreau JJ. Bounded variation in time. In: Panagiotopoulos PD, Strang G, editors. Topics in nonsmooth mechanics, vol. 1; 1987. p. 1-74.

[33] Moreau JJ. Unilateral contact and dry friction in finite freedom dynamics. In: Moreau JJ, Panagiotopoulos P, editors. Non-smooth mechanics and applications. CISM courses and lectures, vol. 302. Springer; 1988. pp. 1-82.

[34] Moreau JJ. Some numerical methods in multibody dynamics: application to granular materials. Euro J Mech A/Solids 1994;13:93-114.

[35] Munoz J, Bonet J, Huerta A, Peraire J. Upper and lower bounds in limit analysis: adaptive meshing strategies and discontinuous loading. Int J Numer Meth Eng 2009;77:471-501.

[36] Nielsen J. Pressures from flowing granular solids in silos. Philos Trans R Soc A 1998;356:2667-84.

[37] Nouguier-Lehon C, Cambou B, Vincens E. Influence of particle shape and angularity on the behavior of granular materials: a numerical analysis. Int J Numer Anal Meth Geomech 2003;27:1207-26.

[38] Pang JS, Trinkle JC. Complementarity formulations and existence of solutions of dynamic multi-rigid-body contact problems with coulomb friction. Mathe Program 1996;73:199-226.

[39] Petraa C, Gavreab B, Anitescu M, Potraa F. A computational study of the use of an optimization-based method for simulating large multibody systems. Optimiz Meth Softw 2009;24:871-94.

[40] Radjai F, Michel J, Moreau JJ, Roux S. Force distributions in dense twodimensional granular systems. Phys Rev Lett 1996;77:274-7.

[41] Radjai F, Richefeu V. Contact dynamics as a nonsmooth discrete element method. Mech Mater 2009;41:715-28.

[42] Renouf M, Dubois F, Alart P. A parallel version of the non smooth contact dynamics algorithm applied to the simulation of granular media. J Comput Appl Mathe 2004;168:375-82.
[43] Ries A, Wolf DE, Unger T. Shear zones in granular media: three-dimensional contact dynamics simulation. Phys Rev E 2007:195.

[44] Saussine G, Cholet C, Gautier PE, Dubois F, Bohatier C, Moreau JJ. Modelling ballast behaviour under dynamic loading part 1: a 2D polygonal discrete element method approach. Comput Meth Appl Mech Eng 2006;195: 2841-59.

[45] Staron L, Hinch EJ. Study of the collapse of granular columns using twodimensional discrete-grain simulation. J Fluid Mech 2005;545:1-27.

[46] Staron L, Hinch EJ. The spreading of a granular mass: role of grain properties and initial conditions. Granul Matt 2007;9:205-17.

[47] Stegmann T, Török J, Brendel L, Wolf DE. Minimal dissipation theory and shear bands in biaxial tests. Granul Matt 2011;13:565-72.

[48] Stewart DE, Trinkle JC. An implicit time-stepping scheme for rigid body dynamics with coulomb friction. Int J Numer Anal Meth Eng 1996;39: 2673-91.

[49] Sturm JF. SeDuMi 1.02, a MATLAB toolbox for optimizing over symmetric cones. Optim Meth Softw 1999:625-53. http://sedumi.mcmaster.ca/.

[50] Taboada A, Chang KJ, Radjai F, Bouchette F. Rheology force transmission and shear instabilities in frictional granular media from biaxial numerical test using the contact dynamics method. J Geophys Res 2005;110:1-24.

[51] Tasora A, Anitescu M. A convex complementarity approach for simulating large granular flows. J Comput Nonlin Dynam 2010;5:031004.

[52] Tasora A, Anitescu M. A matrix-free cone complementarity approach for solving large-scale, nonsmooth, rigid body dynamics. Comput Meth Appl Mech Eng 2011;200:439-53.

[53] Thornton C. Numerical simulations of deviatoric shear deformation of granular media. Geotechnique 2000;50:43-53.

[54] da Silva M Vicente, Antao AN. A nonlinear programming method approach for upper bound limit analysis. Int J Numer Meth Eng 2007;72:1192-218.

[55] da Silva M Vicente, Antao AN. Upper bound limit analysis with a parallel mixed finite element formulation. Int J Solids Struct 2008;45:5788-804.

[56] da Silva M Vicente, Antao AN. A novel augmented Lagrangian-based formulation for upper-bound limit analysis. Int J Numer Meth Eng 2012;89(12):1471-96.

[57] Wood WL. Practical time-stepping schemes. Oxford University Press; 1990.

[58] Zenit R. Computer simulations of the collapse of a granular column. Phys Fluids 2005;17:031703.

[59] Zouain N, Herskovits J, Borges LA, Feijóo RA. An iterative algorithm for limit analysis with nonlinear yield functions. Int J Solids Struct 1993;30(10): :1397-1417.

[60] Zuriguel I, Mullin T, Rotter JM. Effect of particle shape on the stress dip under a sandpile. Phys Rev Lett 2007;98:028001. 